\title{
Complexity in Linear Systems: A Chaotic Linear Operator on the Space of Odd $2 \pi$-Periodic Functions
}

\author{
Tamás Kalmár-Nagy ${ }^{1}$ and Márton Kiss ${ }^{2}$ \\ ${ }^{1}$ Department of Fluid Mechanics, Faculty of Mechanical Engineering, Budapest University of Technology and Economics, \\ Budapest, Hungary \\ ${ }^{2}$ Institute of Mathematics, Faculty of Natural Sciences, Budapest University of Technology and Economics, Budapest, Hungary \\ Correspondence should be addressed to Tamás Kalmár-Nagy; mpe@kalmarnagy.com
}

Received 28 July 2016; Revised 6 December 2016; Accepted 28 December 2016; Published 22 February 2017

Academic Editor: Sylvain Sené

Copyright (c) 2017 Tamás Kalmár-Nagy and Márton Kiss. This is an open access article distributed under the Creative Commons Attribution License, which permits unrestricted use, distribution, and reproduction in any medium, provided the original work is properly cited.

\begin{abstract}
Not just nonlinear systems but infinite-dimensional linear systems can exhibit complex behavior. It has long been known that twice the backward shift on the space of square-summable sequences $l_{2}$ displays chaotic dynamics. Here we construct the corresponding operator $\mathscr{C}$ on the space of $2 \pi$-periodic odd functions and provide its representation involving a Principal Value Integral. We explicitly calculate the eigenfunction of this operator, as well as its periodic points. We also provide examples of chaotic and unbounded trajectories of $\mathscr{C}$.
\end{abstract}

\section{Introduction}

Linear systems have commonly been thought to exhibit relatively simple behavior. Surprisingly, infinite-dimensional linear systems can have complex dynamics. In particular, Rolewicz in 1969 [1] showed that the backward shift $B$ multiplied by 2 (i.e., $2 B$ ) on the space of square-summable sequences $l_{2}$ exhibits transitivity (and thus gives rise to chaotic dynamics). A nice exposition of dynamics of infinite-dimensional operators is given in $[2,3]$ and the recent books $[4,5]$. While chaoticity of linear operators is at first puzzling and the backward shift example seems contrived, these operators are not rare. In fact, Herrero [6] and Chan [7] showed that chaotic linear operators are dense (with respect to pointwise convergence) in the set of bounded linear operators. In addition to $2 B$ there are many examples of chaotic linear operators including weighted shifts [8], composition operators [9], and differentiation and translations [10-12]. It has also been argued in $[13,14]$ that nonlinearity is not necessarily required for complex behavior; an infinite-dimensional state space can also provide the ingredients of chaotic dynamics.

Several recent papers explore chaotic behavior of linear systems (see, e.g., [15, 16]). Bernardes et al. [17], for example, obtain new characterizations of Li-Yorke chaos for linear operators on Banach and Fréchet spaces.

Here we construct a chaotic linear operator by "lifting" $2 B$ to the space $L_{2}$ of square-integrable functions (more precisely to the Hilbert space $L_{2}(0, \pi)$ of $2 \pi$-periodic odd functions). Our main tool in finding the expression for the backward shift is utilizing a smidgen of distribution theory and Cauchy's principal value, a method for obtaining a finite result for a singular integral. The principal value (PV) integral (see, e.g., [18], p. 457) of a function $f$ about a point $c \in[a, b]$ is given by

$$
\begin{aligned}
\mathrm{PV} & \int_{a}^{b} f(x) d x \\
& =\lim _{\varepsilon \rightarrow 0+}\left(\int_{a}^{c-\varepsilon} f(x) d x+\int_{c+\varepsilon}^{b} f(x) d x\right) .
\end{aligned}
$$

The PV integral is commonly used in many fields of physics. A review of developments in the mathematics and methods for Principal Value Integrals is presented in [19]. Cohen et al. [20] examine first-order PV integrals and analyze several of their important properties. The structure of the paper is the following. In Section 2 we relate the backward shift on $l_{2}$ to a shift on $L_{2}(0, \pi)$. We state and prove a theorem 
about expressing this shift on $L_{2}(0, \pi)$ in terms of a PV integral. In Section 3 we define and analyze the corresponding chaotic operator $\mathscr{C}$ on $L_{2}(0, \pi)$, including finding its eigenvectors and periodic points. We provide examples of unbounded and chaotic trajectories of $\mathscr{C}$. In Section 4 we draw conclusions. We also show that utilizing the representation of operator $\mathscr{C}$ one can obtain principal values of certain integrals.

\section{A Chaotic Linear Operator on the Space of $2 \pi$-Periodic Odd Functions}

The backward shift $B$ on the infinite-dimensional Hilbert space $l_{2}$ of square-summable sequence is defined as

$$
B_{a}=\left(a_{2}, a_{3}, \ldots\right),
$$

where $a=\left(a_{1}, a_{2}, \ldots\right)$, such that $\sum_{n=0}^{\infty}\left|a_{n}\right|^{2}<\infty$. The Hilbert space $L_{2}(0, \pi)$ of square-integrable functions is isomorphic with $l_{2}$ (by the Riesz-Fischer theorem) and is a natural functional representation of the sequence space $l_{2}$. By odd extension, elements of $L_{2}(0, \pi)$ can be viewed as odd $2 \pi$-periodic square-integrable functions so that $L_{2}(0, \pi)$ is also isomorphic with the space of odd $2 \pi$-periodic squareintegrable functions. Now we "lift" $a \in l_{2}$ to $L_{2}(0, \pi)$ by the summation

$$
f(t)=\sum_{n=1}^{\infty} a_{n} \sin n t
$$

Clearly, the $n$th Fourier coefficient of $f(t)$ is expressed as

$$
a_{n}=\frac{2}{\pi} \int_{0}^{\pi} f(\xi) \sin n \xi d \xi
$$

We define the backward shift $\mathscr{B}$ acting on $L_{2}(0, \pi)$ as

$$
\mathscr{B} f(t)=\sum_{n=1}^{\infty} a_{n+1} \sin n t=\sum_{n=1}^{\infty} a_{n} \sin (n-1) t .
$$

Therefore

$$
\begin{aligned}
\mathscr{B} f(t) & =\frac{2}{\pi} \sum_{n=1}^{\infty}\left(\int_{0}^{\pi} f(\xi) \sin (n+1) \xi d \xi\right) \sin n t \\
& =\frac{2}{\pi} \sum_{n=1}^{\infty}\left(\int_{0}^{\pi} f(\xi) \sin n \xi d \xi\right) \sin (n-1) t .
\end{aligned}
$$

Our main result is the following.

Theorem 1. $\mathscr{B} f(t)$ can be expressed as

$$
\mathscr{B} f(t)=f(t) \cos t-\frac{1}{\pi} \mathrm{PV} \int_{0}^{\pi} \frac{\sin t \sin \xi}{\cos t-\cos \xi} f(\xi) d \xi .
$$

The strategy of the proof is the following: let us denote by $\mathscr{A} f(t)$ the right-hand side of (7) and by $P_{n}$ the projection from $L_{2}(0, \pi)$ onto the linear span of $\{\sin t, \sin 2 t, \ldots, \sin n t\}$. The sequence $\mathscr{B} P_{n}$ converges strongly to $B$. In particular, for every $\varphi \in \mathscr{D}(0, \pi)$ (this is the space of test functions, see
Definition 2 in the Appendix), $\mathscr{B} P_{n} \varphi \rightarrow \mathscr{B} \varphi$ in $L_{2}(0, \pi)$. Then a subsequence tends to $\mathscr{B} \varphi$ almost everywhere. Hence if we prove that $\mathscr{B} P_{n} \varphi(t)$ tends to $\mathscr{A} \varphi(t)$ for all fixed $t$, then $\mathscr{A} \varphi=\mathscr{B} \varphi$ almost everywhere as functions in $L_{2}(0, \pi)$; that is, $\mathscr{A}=\mathscr{B}$ on $\mathscr{D}(0, \pi)$. Finally, $\mathscr{D}(0, \pi)$ is a dense set in $L_{2}(0, \pi)$; thus $\mathscr{A}=\mathscr{B}$ on the whole space $L_{2}(0, \pi)$.

Proof. We start from

$$
\begin{aligned}
\mathscr{B} P_{n} f(t) & =\frac{2}{\pi} \sum_{k=1}^{n}\left(\int_{0}^{\pi} f(\xi) \sin k \xi d \xi\right) \sin (k-1) t \\
& =\frac{2}{\pi} \int_{0}^{\pi} f(\xi)\left(\sum_{k=1}^{n} \sin k \xi \sin (k-1) t\right) d \xi .
\end{aligned}
$$

We first rewrite the "kernel" of (8) as

$$
\begin{aligned}
\sin k \xi & \sin (k-1) t \\
= & \sin k \xi \sin k t \cos t-\sin k \xi \cos k t \sin t \\
= & \frac{\cos k(\xi-t)-\cos k(\xi+t)}{2} \cos t \\
& -\frac{\sin k(\xi-t)+\sin k(\xi+t)}{2} \sin t
\end{aligned}
$$

For test functions $\varphi \in \mathscr{D}(0, \pi)$ and $t \in(0, \pi)$ we get

$$
\begin{aligned}
& \mathscr{B} P_{n} \varphi(t)=\frac{1}{\pi} \cos t \\
& \cdot \int_{0}^{\pi} \varphi(\xi) \sum_{k=1}^{n}(\cos k(\xi-t)-\cos k(\xi+t)) d \xi-\frac{1}{\pi} \\
& \cdot \sin t \int_{0}^{\pi} \varphi(\xi) \sum_{k=1}^{n}(\sin k(\xi-t)+\sin k(\xi+t)) d \xi .
\end{aligned}
$$

Taking $n \rightarrow \infty$ limit and utilizing (A.1) and (A.2) yield

$$
\begin{aligned}
\lim _{n \rightarrow \infty} \mathscr{B} P_{n} \varphi(t) & =\cos t\langle(\delta(\xi-t)-\delta(\xi+t)), \varphi(\xi)\rangle \\
- & \frac{1}{2 \pi} \sin t\left\langle\left(\mathscr{P} \cot \frac{\xi-t}{2}+\mathscr{P} \cot \frac{\xi+t}{2}\right), \varphi(\xi)\right\rangle .
\end{aligned}
$$

Since

$$
\begin{aligned}
\cot & \frac{\xi-t}{2}+\cot \frac{\xi+t}{2} \\
& =2 \frac{\sin ((\xi-t) / 2+(\xi+t) / 2)}{2 \sin ((\xi-t) / 2) \sin ((\xi+t) / 2)} \\
& =\frac{2 \sin \xi}{\cos t-\cos \xi},
\end{aligned}
$$

the limit calculated in (11) is the same as $\mathscr{A} f(t)$.

Our "chaotic" operator (twice the backward shift) is now defined as

$$
\begin{aligned}
\mathscr{C} f(t) & =2 \mathscr{B} f(t) \\
& =2 f(t) \cos t-\frac{2}{\pi} \mathrm{PV} \int_{0}^{\pi} \frac{\sin t \sin \xi}{\cos t-\cos \xi} f(\xi) d \xi .
\end{aligned}
$$




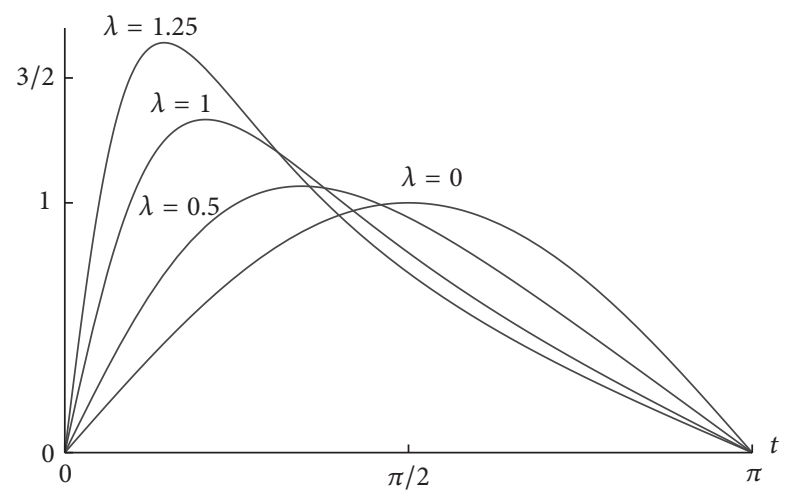

FIGURE 1: Eigenfunctions of $\mathscr{C}$ for $\lambda=0,0.5,1$, and 1.25 .

\section{Analysis of $\mathscr{C}$}

The eigenfunctions of $\mathscr{C}$ can be found from the eigenvalue relation

$$
\mathscr{C} f^{*}(t)=\lambda f^{*}(t) .
$$

Instead of using (13), we revert to (5) to write

$$
\sum_{n=1}^{\infty} 2 a_{n+1} \sin n t=\sum_{n=1}^{\infty} \lambda a_{n} \sin n t .
$$

From this we have

$$
a_{n+1}=\left(\frac{\lambda}{2}\right) a_{n}
$$

and thus

$$
f^{*}(t)=a_{1} \sum_{n=1}^{\infty}\left(\frac{\lambda}{2}\right)^{n-1} \sin n t=a_{1} \frac{4 \sin t}{4+\lambda^{2}-4 \lambda \cos t} .
$$

The functions corresponding to eigenvalue $\lambda=1$ are

$$
f^{*}(t)=a_{1} \frac{4 \sin (t)}{5-4 \cos (t)} ;
$$

that is, the functions $a_{1}(4 \sin (t) /(5-4 \cos (t)))$ are left invariant under the action of $\mathscr{C}$. In other words $f^{*}(t)$ 's are fixed points of operator $\mathscr{C}$. A family of eigenfunctions is displayed in Figure 1 (we set $a_{1}=1$ ).

To better characterize the action of $\mathscr{C}$ we want to understand how a given function is "shaped" under the repeated application of $\mathscr{C}$. For $f \in L_{2}(0, \pi)$ the orbit of $f$ is defined as $\operatorname{Orb}(\mathscr{C}, f)=\left\{f, \mathscr{C} f, \mathscr{C}^{2} f, \ldots\right\}$, where $\mathscr{C}^{k}=\underbrace{\mathscr{C} \circ \ldots \circ \mathscr{C}}_{k}$ is the $k$ th composition of $\mathscr{C}$ with itself. The $k$-fold composition acts on $f(t)$ as

$$
\mathscr{C}^{k} f(t)=2^{k} \sum_{n=1}^{\infty} a_{n+k} \sin n t
$$

A given $f \in L_{2}(0, \pi)$ is a $T$-periodic point of $\mathscr{C}$ if $\mathscr{C}^{T} f=f$ for some $T \geq 1$ (a fixed point is a 1-periodic point; i.e., $T=1$ ).
We are now in the position to construct $T$-periodic points of $\mathscr{C}$.

Introducing $y=\left\{y_{1}, \ldots, y_{T}\right\}$, a $T$-periodic point of $2 B$ (acting on $l_{2}$ ) can be written as [3]

$$
a=\left(y_{1}, \ldots, y_{T}, \frac{y_{1}}{2^{T}}, \ldots, \frac{y_{T}}{2^{T}}, \frac{y_{1}}{2^{2 T}}, \ldots, \frac{y_{T}}{2^{2 T}}, \ldots\right),
$$

whose $n$th component is given by

$$
a_{n}=\frac{y_{(n-1 \bmod T)+1}}{2^{[(n-1) / T] T}} .
$$

Using the linearity of $\mathscr{C}$ we can easily find a period-2 point of $\mathscr{C}$, that is, a function $g(t)$ such that $\mathscr{C}^{2} g(t)=g(t)$ :

$$
\begin{aligned}
g(t) & =y_{1} \sum_{n=1}^{\infty} \frac{\sin (2 n-1) t}{2^{2 n-2}}+y_{2} \sum_{n=1}^{\infty} \frac{\sin (2 n) t}{2^{2 n-2}} \\
& =y_{1} \frac{20 \sin t}{17-8 \cos 2 t}+y_{2} \frac{16 \sin 2 t}{17-8 \cos 2 t} .
\end{aligned}
$$

In general, we find a period- $T$ point of $\mathscr{C}$ as

$$
\begin{aligned}
& f(t)=y_{1}\left(\sin t+\frac{\sin (T+1) t}{2^{T}}+\cdots+\frac{\sin (n T+1) t}{2^{n T}}\right. \\
& +\cdots) \\
& +y_{2}\left(\sin 2 t+\frac{\sin (T+2) t}{2^{T}}+\cdots+\frac{\sin (n T+2) t}{2^{n T}}\right. \\
& \quad+\cdots)+\cdots+ \\
& +y_{T}\left(\sin T t+\frac{\sin (2 T) t}{2^{T}}+\cdots+\frac{\sin ((n+1) T) t}{2^{n T}}\right. \\
& +\cdots) .
\end{aligned}
$$

By defining the "basis functions"

$$
\begin{aligned}
\phi(l, T) & =\sum_{n=1}^{\infty} \frac{\sin ((n-1) T+1+l) t}{2^{(n-1) T}} \\
& =\frac{2^{T}\left(2^{T} \sin (l+1) t+\sin ((T-1-l) t)\right)}{1+4^{T}-2^{T+1} \cos T t},
\end{aligned}
$$

a period- $T$ point of $\mathscr{C}$ can be expressed as the linear combination

$$
f(t)=\sum_{l=1}^{T} y_{l} \phi(l-1, T) .
$$



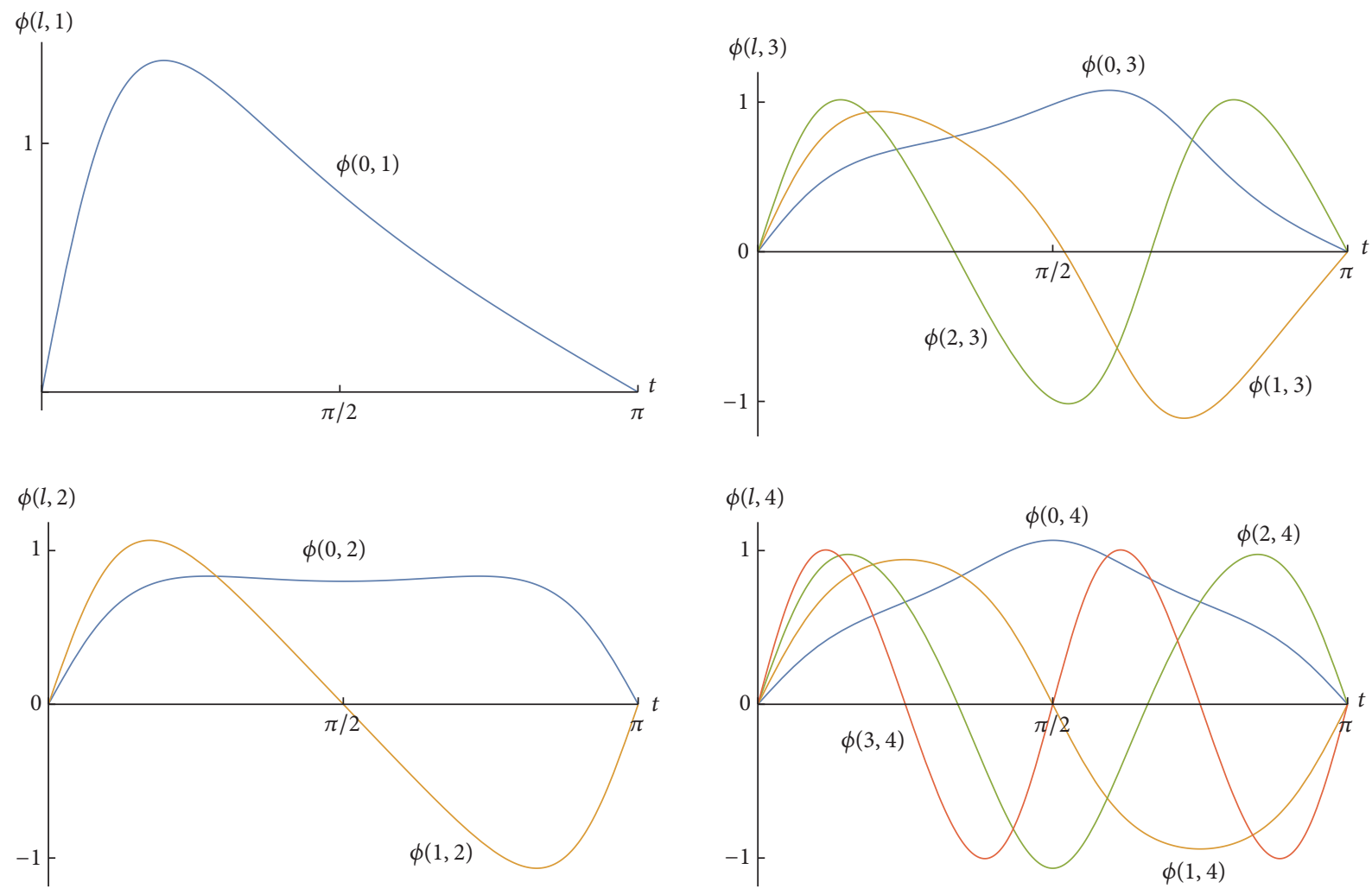

Figure 2: Basis functions $\phi(l, T)$ for $T=1, \ldots, 4$ and $l=0, \ldots, T-1$.

The first few basis functions are (shown in Figure 2)

$$
\begin{aligned}
& \phi(0,1)=\frac{4 \sin (t)}{5-4 \cos (t)} \quad T=1, \\
& \phi(0,2)=\frac{20 \sin (t)}{17-8 \cos (2 t)}, \\
& \phi(1,2)=\frac{16 \sin (2 t)}{17-8 \cos (2 t)}, \quad T=2, \\
& \phi(0,3)=\frac{64 \sin (t)+8 \sin (2 t)}{65-16 \cos (3 t)}, \\
& \phi(1,3)=\frac{8 \sin (t)+64 \sin (2 t)}{65-16 \cos (3 t)}, \\
& \phi(2,3)=\frac{64 \sin (3 t)}{65-16 \cos (3 t)}, T=3, \\
& \phi(0,4)=\frac{256 \sin (t)+16 \sin (3 t)}{257-32 \cos (4 t)}, \\
& 272 \sin (2 t) \\
& 257-32 \cos (4 t)
\end{aligned}
$$

$$
\begin{aligned}
& \phi(2,4)=\frac{16 \sin (t)+256 \sin (3 t)}{257-32 \cos (4 t)}, \\
& \phi(3,4)=\frac{256 \sin (4 t)}{257-32 \cos (4 t)}
\end{aligned}
$$$$
T=4
$$

Now we turn to creating a function that gives rise to a chaotic orbit under the action of $\mathscr{C}$. First, we note that for $2 B$ (on $l_{2}$ ) the point

$$
y=\left(\frac{y_{1}}{1}, \frac{y_{2}}{2}, \frac{y_{3}}{4}, \ldots\right),
$$

where $y_{i}$ is the $i$ th digit of a normal irrational number (whose digits are uniformly distributed) and generates a chaotic orbit. $\pi$ is believed to be normal, so we take $y_{i}$ to be the $i$ th digit of $\pi$. We now lift this point to $L_{2}(0, \pi)$ using (3):

$$
\Psi(t)=\sum_{i=1}^{\infty} \frac{y_{i}}{2^{i-1}} \sin n t .
$$

Figure 3 shows the first 10 elements of the orbit of $\Psi$ under the action of $\mathscr{C}$, that is, $\operatorname{Orb}(\mathscr{C}, \Psi)$. The first element of the orbit is $\Psi$ itself.

Figure 4 shows the orbit $\operatorname{Orb}(\mathscr{C}, \Psi)$ evaluated at three different $t$ 's $(\pi / 20, \pi / 2,19 \pi / 20)$ for 200 iterations.

Engineering applications of chaotic orbits include design of fuel efficient space missions [21] and efficient mixing protocols for microfluids [22]. 


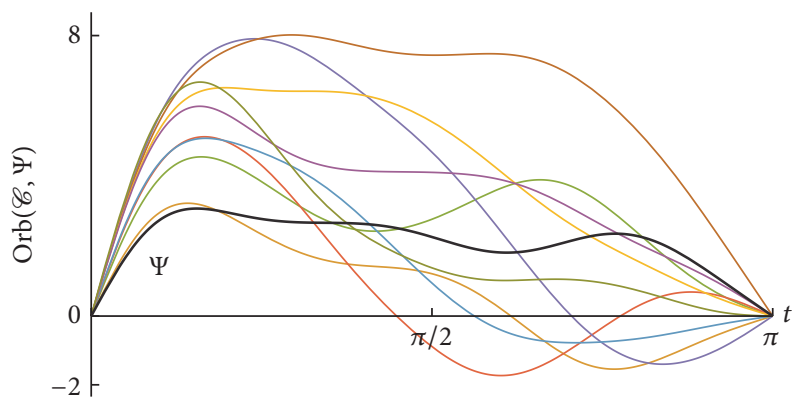

Figure 3: The first 10 elements of $\operatorname{Orb}(\mathscr{C}, \Psi)$.

Now we examine the effect of $\mathscr{C}$ on some commonly used periodic functions, namely, the ramp, the square-wave, and the triangle. The Fourier series of these functions are the following:

$$
\begin{aligned}
& \operatorname{Ramp}(t)=t=\sum_{k=1}^{\infty} \frac{2(-1)^{k+1}}{k} \sin k t \quad(-\pi<t<\pi), \\
& \operatorname{Sqw}(t)= \begin{cases}-\pi & -\pi<t<0 \\
\pi & 0<t<\pi\end{cases} \\
& =\sum_{k=1}^{\infty} \frac{2}{k} \sin ^{2}\left(\frac{k \pi}{2}\right) \sin k t \\
& =\sum_{k=1}^{\infty} \frac{2}{2 k+1} \sin (2 k+1) t \text {, } \\
& \text { Triangle }(t)= \begin{cases}t & 0<t<\frac{\pi}{2} \\
\frac{\pi}{2}-t & \frac{\pi}{2}<t<\pi\end{cases} \\
& =\sum_{k=1}^{\infty} \frac{4}{k^{2} \pi} \sin \left(\frac{k \pi}{2}\right) \sin k t \\
& =\sum_{k=1}^{\infty} \frac{4(-1)^{k+1}}{(2 k+1)^{2} \pi} \sin (2 k+1) t \text {. }
\end{aligned}
$$

Figure 5 shows the first 4 elements of the orbits of these functions. First, we note that the norm of the iterates grows (moreover, each Fourier coefficient tends to infinity); that is, these functions have unbounded orbits under the action of $\mathscr{C}$. Second, the graphs of the even iterates $\left(\mathscr{C}^{2 n}\right)$ of $\operatorname{Ramp}(t), \operatorname{Sqw}(t)$, and Triangle $(t)$ are similar to the graph of $\tan (t / 2),-\tan (t+\pi / 2)$, and $\pm(-1)^{[t / \pi-1 / 2]} \tan t$, respectively. This is not too surprising, since the Fourier expansion of $\tan (t / 2)$ is $2 \sum_{k=1}^{\infty}(-1)^{k+1} \sin k t$ which is close in some sense to

$$
\frac{2 n}{2^{2 n}} \mathscr{C}^{2 n} \operatorname{Ramp}(t)=2 \sum_{k=1}^{\infty}(-1)^{k+1}\left(1-\frac{k}{2 n+k}\right) \sin k t
$$
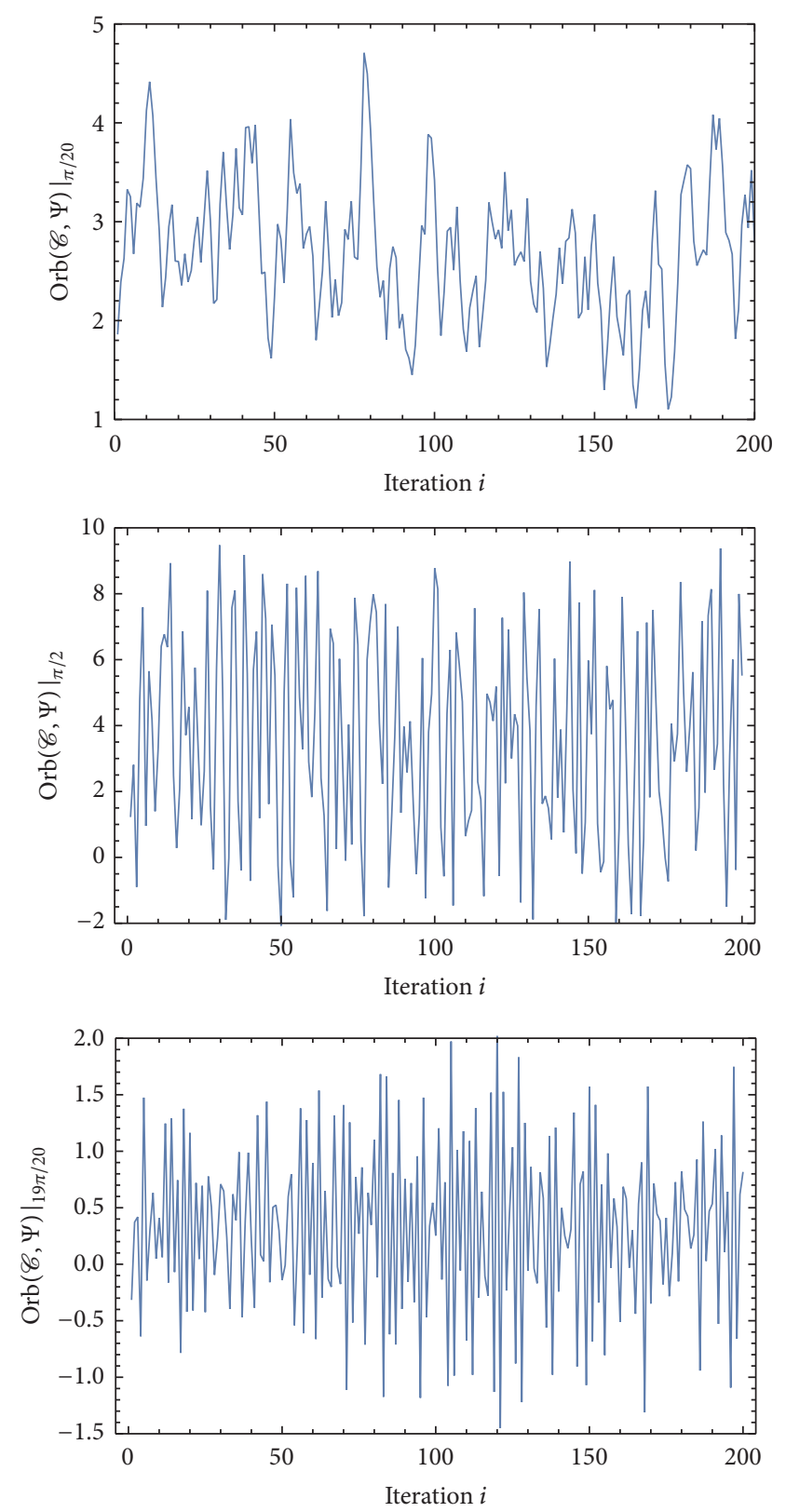

Figure 4: The orbit $\operatorname{Orb}(\mathscr{C}, \Psi)$ for 200 iterations evaluated at $t=$ $\pi / 20, \pi / 2,19 \pi / 20$.

for large enough $n$. Unbounded orbits of differential equations (the so-called escape orbits) play an important role in Newtonian gravitation [23].

\section{Conclusions}

Contrary to common belief, linear systems can display complicated dynamics. Starting from twice the backward shift on $l_{2}$ we constructed the corresponding shift operator $C$ on $L_{2}(0, \pi)$ (the space of odd, $2 \pi$-periodic functions) and provided its representation using a modicum of distribution theory and Cauchy's Principal Value Integral. We explicitly calculated the periodic points of the operator (including its 

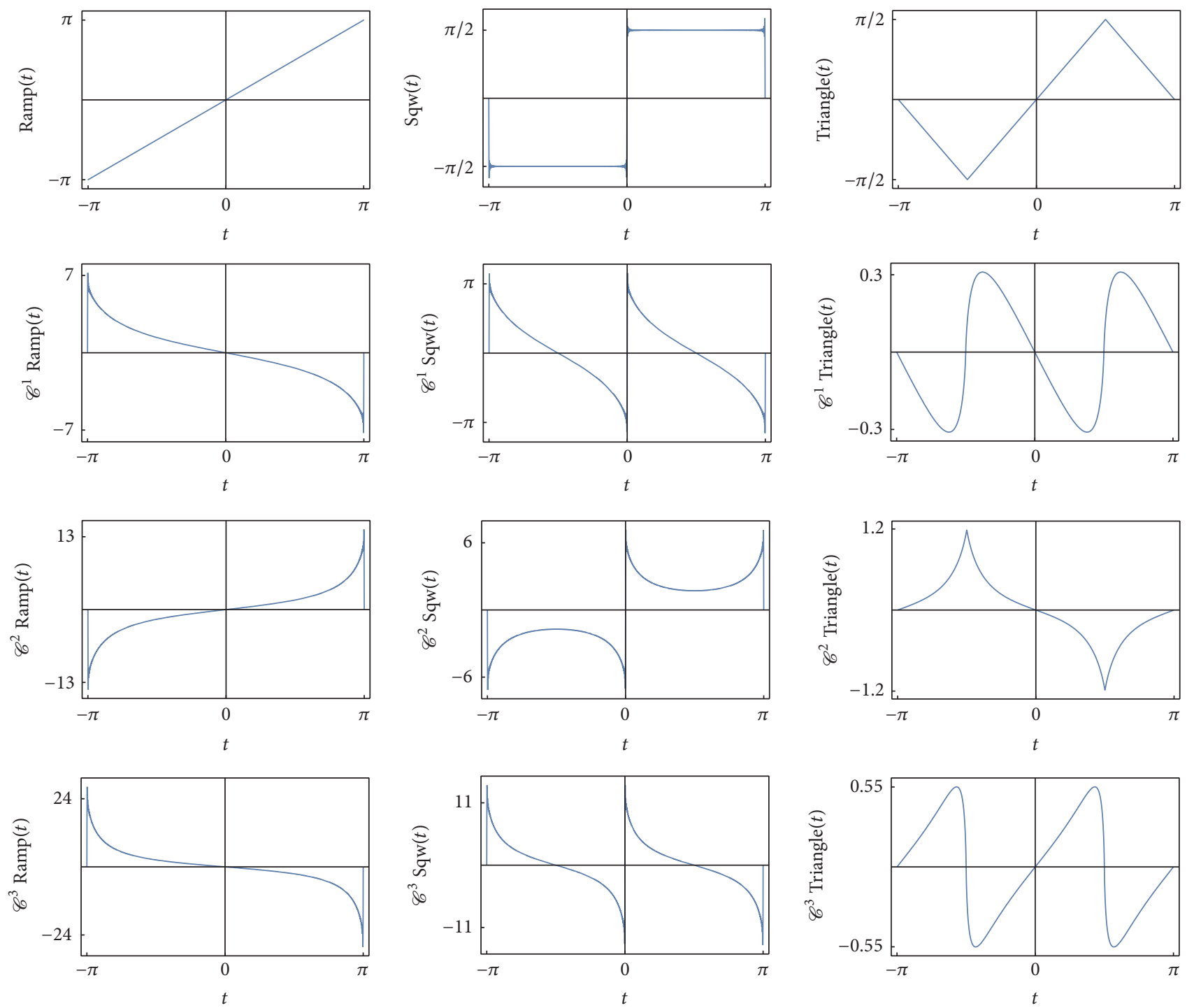

FIGURE 5: Iterates of the ramp, square-wave, and triangle functions.

nontrivial fixed point) and provided examples of chaotic and unbounded trajectories of $\mathscr{C}$.

We note here that utilizing representation (7) of operator $\mathscr{C}$ one can actually calculate principal values. To wit, rearranging (7) yields

$$
\begin{aligned}
\mathrm{PV} & \int_{0}^{\pi} \frac{\sin t \sin \xi}{\cos t-\cos \xi} f(\xi) d \xi \\
& =\pi f(t) \cos t-\frac{\pi}{2} \mathscr{C} f(t) .
\end{aligned}
$$

For the simplest case, when $f(t)$ is the eigenfunction of operator $\mathscr{C}$, that is, $\mathscr{C} f(t)=\lambda f(t)$, we have (cf. (17))

$$
\begin{aligned}
\mathrm{PV} & \int_{0}^{\pi} \frac{\sin t \sin \xi}{\cos t-\cos \xi} \frac{4 \sin \xi}{4+\lambda^{2}-4 \lambda \cos \xi} d \xi \\
& =\frac{2 \pi \sin t(2 \cos t-\lambda)}{4+\lambda^{2}-4 \lambda \cos t} .
\end{aligned}
$$

The basis functions $\phi(l, T)$ can similarly be used to obtain $\mathrm{PV}$ integrals. The Principal Value Integral is a tool commonly used in physics, but not in engineering-related fields. We hope that this connection between chaotic operators and Principal Value Integrals will stimulate further research.

\section{Appendix}

In this section we give a short introduction on distributional derivatives. The following definitions together with Proposition 5 can be found in standard textbooks on partial differential equations; see, for example, [24, 25].

Notation 1 (multi-index). Let $\Omega$ be an open subset of $\mathbb{R}^{n}$ and let $\varphi: \Omega \rightarrow \mathbb{R}$ be a smooth function. Then, for a vector $\alpha=\left(\alpha_{1}, \alpha_{2}, \ldots, \alpha_{n}\right), \partial^{\alpha} \varphi$ denotes the partial derivative $\partial_{1}^{\alpha_{1}} \partial_{2}^{\alpha_{2}} \cdots \partial_{n}^{\alpha_{n}} \varphi$ of order $|\alpha|=\alpha_{1}+\alpha_{2}+\cdots+\alpha_{n}$. 
Definition 2 (test functions). Let $\mathscr{D}(\Omega)$ denote the space of test functions on the open set $\Omega \subset \mathbb{R}^{n}$, that is, $\mathscr{D}(\Omega)=$ $C_{c}^{\infty}(\Omega)$, endowed with the following convergence: $\varphi_{n} \rightarrow \varphi$ if there is a compact subset $K$ of $\Omega$ containing the support of $\varphi_{n}$ for all $n$ and for every multi-index $\alpha \partial^{\alpha} \varphi_{n} \rightarrow \partial^{\alpha} \varphi$ uniformly.

Definition 3 (distributions). Let $\mathscr{D}^{\prime}(\Omega)$ denote the space of continuous linear functionals on $\mathscr{D}(\Omega)$. The convergence on $\mathscr{D}(\Omega)$ induces a weak or pointwise convergence on $\mathscr{D}^{\prime}(\Omega)$; namely, $u_{n} \rightarrow u$ if $\left\langle u_{n}, \varphi\right\rangle \rightarrow\langle u, \varphi\rangle$ for all $\varphi \in \mathscr{D}(\Omega)$.

Note that every function $h$ locally integrable on $\Omega$ acts as a distribution via $\langle h, \varphi\rangle=\int_{\Omega} h \varphi$. Then $h$ is called a regular distribution.

Definition 4 (derivatives of distributions). Let $u \in \mathscr{D}^{\prime}(\Omega)$. Then $\left\langle\partial^{\alpha} u, \varphi\right\rangle=(-1)^{|\alpha|}\left\langle u, \partial^{\alpha} \varphi\right\rangle$.

Note the similarity with the integration by parts formula for regular distributions.

Proposition 5. Differentiation is a continuous operation with respect to the pointwise convergence of distributions. As a consequence, derivatives of infinite series of distributions can be calculated by term-by-term differentiation.

To aid the proof of Theorem 1 we state and prove the following.

Proposition 6. Consider the following:

$$
\begin{aligned}
\sum_{k=1}^{\infty} \cos k x & =\pi \sum_{k=-\infty}^{\infty} \delta(x-2 k \pi)-\frac{1}{2}, \\
-\sum_{k=1}^{\infty} \sin k x & =-\frac{1}{2} \mathscr{P} \cot \frac{x}{2},
\end{aligned}
$$

where the distribution $\mathscr{P} \cot (x / 2)$ acts on a function $\varphi$ as

$$
\left\langle\mathscr{P} \cot \frac{x}{2}, \varphi(x)\right\rangle=\sum_{k=-\infty}^{\infty} \mathrm{PV} \int_{(2 k-1) \pi}^{(2 k+1) \pi} \cot \frac{x}{2} \varphi(x) d x
$$

where the principal values are given in the sense of (1) with $a=$ $(2 k-1) \pi, b=(2 k+1) \pi$, and $c=2 k \pi$.

Proof. We start with the identities [26]

$$
\begin{aligned}
& \sum_{k=1}^{\infty} \frac{\sin k x}{k}=\frac{\pi-x}{2} \quad(0<x<2 \pi), \\
& \sum_{k=1}^{\infty} \frac{\cos k x}{k}=-\frac{1}{2} \ln 2(1-\cos x) \quad(0<x<2 \pi) .
\end{aligned}
$$

By periodicity, both equalities extend to $\mathbb{R}$. Term-by-term differentiation of the left hand sides of (A.4) and (A.5) (using Proposition 5) results immediately in the left hand sides of
(A.1) and (A.2). Concerning the right-hand side of (A.4), let us denote the $2 \pi$-periodic extension of $(\pi-x) / 2$ by $h(x)$. Then

$$
\begin{aligned}
\left\langle h^{\prime}(x), \varphi(x)\right\rangle & =-\left\langle h(x), \varphi^{\prime}(x)\right\rangle \\
& =-\int_{-\infty}^{\infty} h(x) \varphi^{\prime}(x) d x \\
& =-\sum_{k=-\infty}^{\infty} \int_{2 k \pi}^{2(k+1) \pi} h(x) \varphi^{\prime}(x) d x .
\end{aligned}
$$

Note that this is actually a finite sum as the test function $\varphi$ is compactly supported. One partial integration in all terms leads to

$$
\begin{aligned}
& -\sum_{k=-\infty}^{\infty} \int_{2 k \pi}^{2(k+1) \pi} h(x) \varphi^{\prime}(x) d x \\
& =-\sum_{k=-\infty}^{\infty}\left\{[h(x) \varphi(x)]_{2 k \pi}^{2(k+1) \pi}\right. \\
& \left.-\int_{2 k \pi}^{2(k+1) \pi}\left(-\frac{1}{2} \varphi(x)\right) d x\right\}=\sum_{k=-\infty}^{\infty} \pi \varphi(2 k \pi) \\
& -\int_{-\infty}^{\infty} \frac{1}{2} \varphi(x) d x,
\end{aligned}
$$

which is the right-hand side of (A.1) applied to $\varphi(x)$. Similarly, let us define $h(x)$ as the $2 \pi$-periodic extension of $-(1 / 2) \ln 2(1-\cos x)$ (the right-hand side of (A.5)). The ordinary derivative of $h(x)$ is $\cot (x / 2)$, but, in contrast with the previous case, this is not locally integrable near the integer multiples of $2 \pi$. Cutting the singularities first and then integrating by parts,

$$
-\sum_{k=-\infty}^{\infty} \int_{2 k \pi}^{2(k+1) \pi} h(x) \varphi^{\prime}(x) d x
$$

$$
\begin{aligned}
& =-\lim _{\varepsilon \rightarrow 0+} \sum_{k=-\infty}^{\infty} \int_{2 k \pi+\varepsilon}^{2(k+1) \pi-\varepsilon} h(x) \varphi^{\prime}(x) d x \\
& =\lim _{\varepsilon \rightarrow 0+} \sum_{k=-\infty}^{\infty} \int_{2 k \pi+\varepsilon}^{2(k+1) \pi-\varepsilon} \cot \frac{x}{2} \varphi(x) d x .
\end{aligned}
$$

This is the same as (A.3), that is, the distribution in (A.2) applied to $\varphi(x)$.

\section{Competing Interests}

The authors declare that they have no competing interests.

\section{Acknowledgments}

This work relates to the scientific program of the project "Development of Quality-Oriented and Harmonized R+D+I Strategy and the Functional Model at BME," supported by the New Hungary Development Plan (Project ID: TÁMOP4.2.1/B-09/1/KMR-2010-0002). The work was also supported 
by the project "Talent Care and Cultivation in the Scientific Workshops of BME" project (Project ID: TÁMOP-4.2.2/B10/1-2010-0009). The authors would like to thank Prof. Tibor Illés for his encouragement of this collaboration.

\section{References}

[1] S. Rolewicz, "On orbits of elements," Studia Mathematica, vol. 32, no. 1, pp. 17-22, 1969.

[2] J. H. Shapiro, Notes on the Dynamics of Linear Operators, Lecture Notes, 2001, http://users.math.msu.edu/users/mshapiro/.

[3] N. S. Feldman, "Linear chaos," 2001, http://home.wlu.edu/ feldmann/Papers/LinearChaos.html.

[4] F. Bayart and É. Matheron, Dynamics of Linear Operators, vol. 179, Cambridge University Press, 2009.

[5] K.-G. Grosse-Erdmann and A. P. Manguillot, Linear Chaos, Springer Science \& Business Media, 2011.

[6] D. A. Herrero, "Hypercyclic operators and chaos," Journal of Operator Theory, vol. 28, no. 1, pp. 93-103, 1992.

[7] K. C. Chan, "The density of hypercyclic operators on a Hilbert space," Journal of Operator Theory, vol. 47, no. 1, pp. 131-143, 2002.

[8] H. N. Salas, "Hypercyclic weighted shifts," Transactions of the American Mathematical Society, vol. 347, no. 3, pp. 993-1004, 1995.

[9] P. Bourdon and J. H. Shapiro, Cyclic Phenomena for Composition Operators, vol. 596, American Mathematical Society, 1997.

[10] R. M. Gethner and J. H. Shapiro, "Universal vectors for operators on spaces of holomorphic functions," Proceedings of the American Mathematical Society, vol. 100, no. 2, pp. 281-288, 1987.

[11] X.-C. Fu and J. Duan, "Infinite-dimensional linear dynamical systems with chaoticity," Journal of Nonlinear Science, vol. 9, no. 2, pp. 197-211, 1999.

[12] F. Martínez-Giménez and A. Peris, "Chaos for backward shift operators," International Journal of Bifurcation and Chaos, vol. 12, no. 8, pp. 1703-1715, 2002.

[13] V. Protopopescu, "Linear vs nonlinear and infinite vs finite: an interpretation of chaos," Tech. Rep. Oak Ridge, Tenn, USA, Oak Ridge National Lab, 1990.

[14] V. Protopopescu and Y. Y. Azmy, "Topological chaos for a class of linear models," Mathematical Models and Methods in Applied Sciences, vol. 2, no. 1, pp. 79-90, 1992.

[15] B. Hou, G. Tian, and S. Zhu, "Approximation of chaotic operators," Journal of Operator Theory, vol. 67, no. 2, pp. 469-493, 2012.

[16] F. Martínez-Giménez, P. Oprocha, and A. Peris, "Distributional chaos for operators with full scrambled sets," Mathematische Zeitschrift, vol. 274, no. 1-2, pp. 603-612, 2013.

[17] J. Bernardes, A. Bonilla, V. Müller, and A. Peris, "Li-yorke chaos in linear dynamics," Ergodic Theory and Dynamical Systems, vol. 35, no. 6, pp. 1723-1745, 2015.

[18] I. N. Bronshtein and K. A. Semendyayev, Handbook of Mathematics, Springer-Verlag, Berlin, Germany, 2013.

[19] K. T. Davies, M. L. Glasser, V. Protopopescu, and F. Tabakin, "The mathematics of principal value integrals and applications to nuclear physics, transport theory, and condensed matter physics," Mathematical Models and Methods in Applied Sciences, vol. 6, no. 6, pp. 833-885, 1996.
[20] S. M. Cohen, K. T. R. Davies, R. W. Davies, and M. H. Lee, "Principal-value integrals-revisited," Canadian Journal of Physics, vol. 83, no. 5, pp. 565-575, 2005.

[21] E. Belbruno, Fly Me to the Moon: An Insider's Guide to the New Science of Space Travel, Princeton University Press, Princeton, NJ, USA, 2007.

[22] C.-Y. Lee, C.-L. Chang, Y.-N. Wang, and L.-M. Fu, "Microfluidic mixing: a review," International Journal of Molecular Sciences, vol. 12, no. 5, pp. 3263-3287, 2011.

[23] F. G. Gascon and D. Peralta-Salas, "Escape to infinity in a Newtonian potential," Journal of Physics A: Mathematical and General, vol. 33, no. 30, pp. 5361-5368, 2000.

[24] V. S. Vladimirov, Methods of the Theory of Generalized Functions, vol. 6 of Analytical Methods and Special Functions, Taylor \& Francis, London, UK, 2002.

[25] R. P. Kanwal, Generalized Functions: Theory and Applications, Springer, Boston, Mass, USA, 3rd edition, 2004.

[26] I. S. Gradshteyn and I. M. Ryzhik, Table of Integrals, Series, and Products, Academic Press, San Diego, Calif, USA, 2014. 


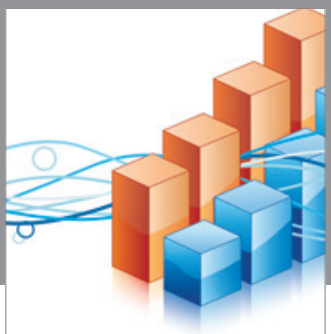

Advances in

Operations Research

vatem alat4

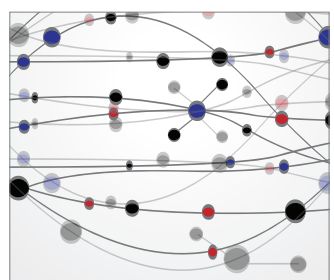

\section{The Scientific} World Journal
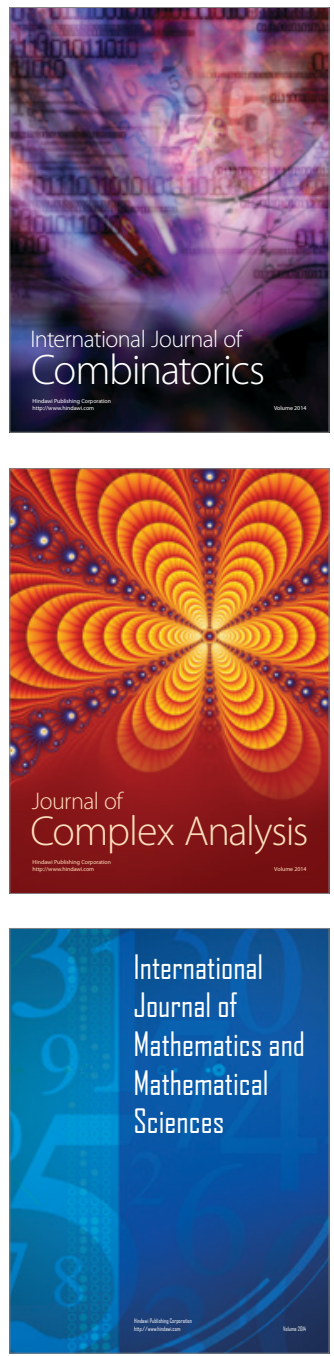
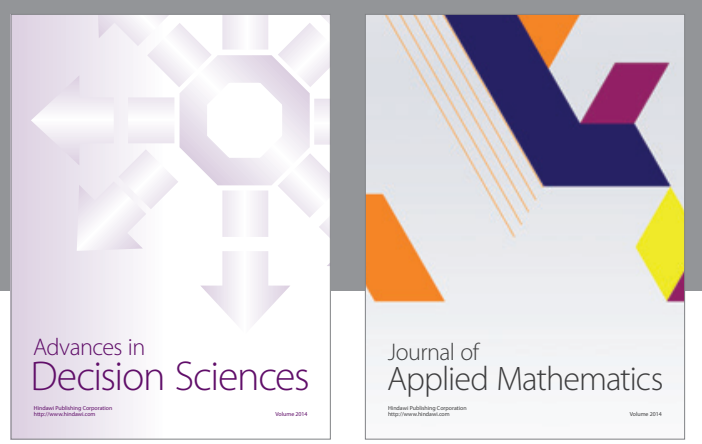

Algebra

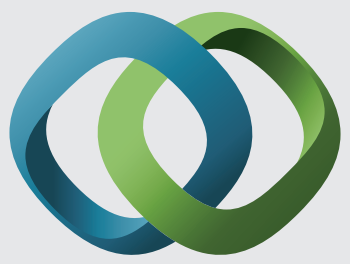

\section{Hindawi}

Submit your manuscripts at

https://www.hindawi.com
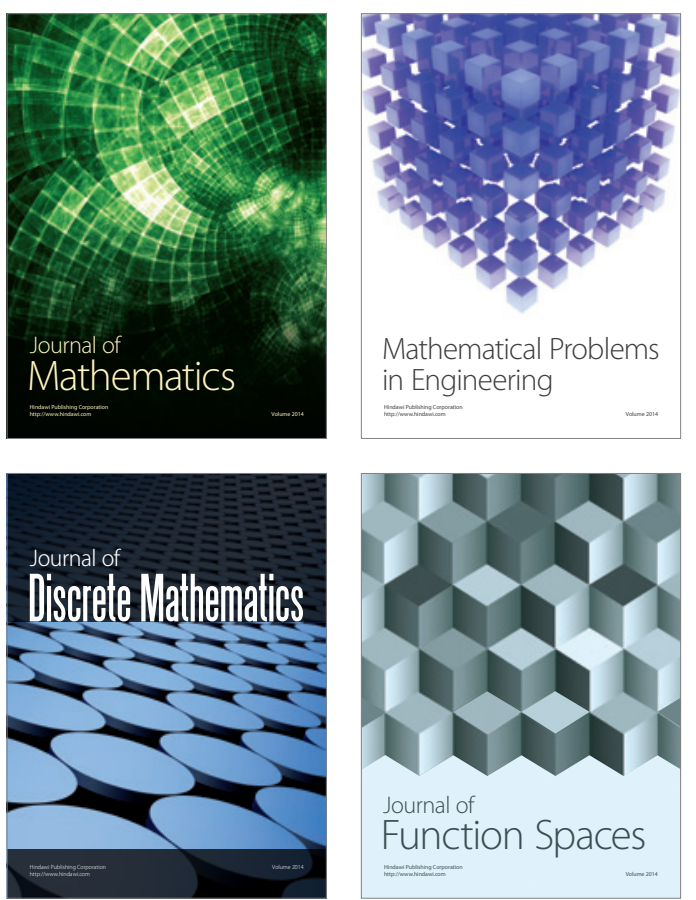

Mathematical Problems in Engineering
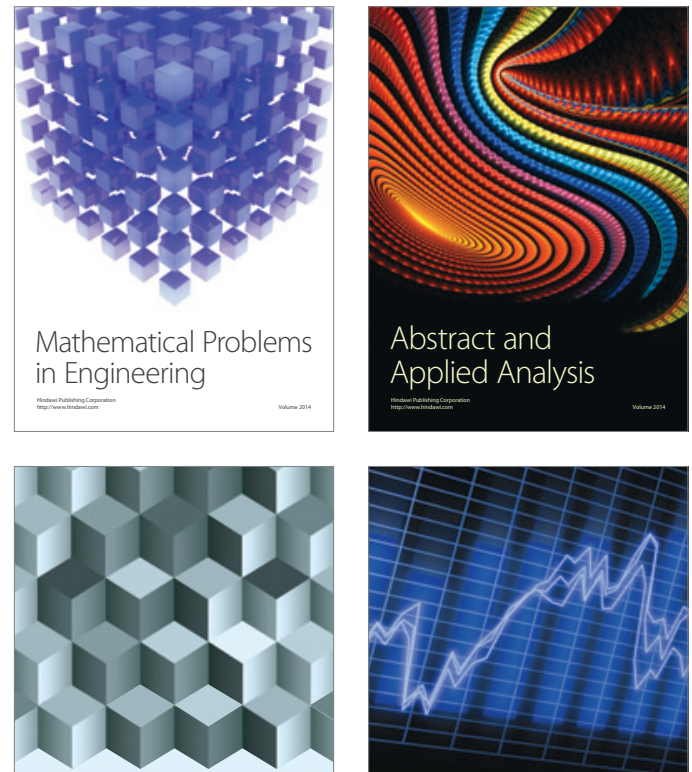

Journal of

Function Spaces

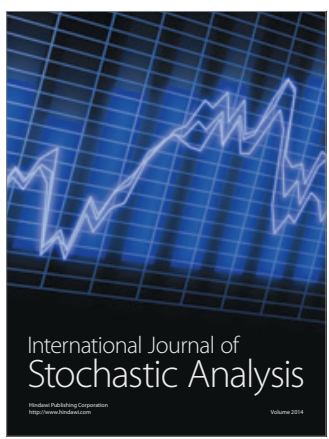

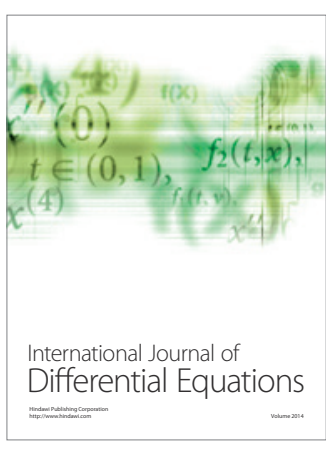
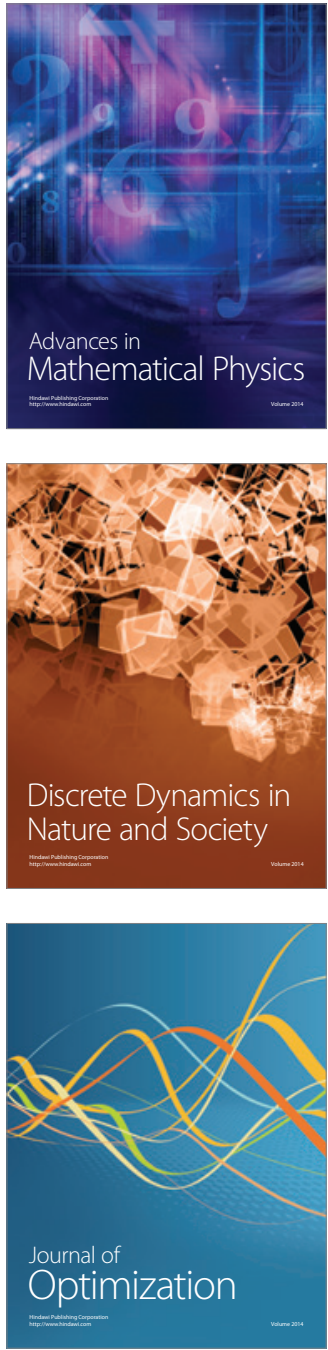